\title{
A New Parallel Method Based on a Genetic Approach for Determination and Classification of Skin Spots
}

\author{
Andrea KUTICS \\ Munehiro DATE \\ The Institute of Physical and Chemical Research (RIKEN), \\ 2-1, Hirosawa, Wako-shi, Saitama 351-01, JAPAN
}

\begin{abstract}
A low cost CCD camera system connected to a transputer network as a parallel processing device has been developed for the determination of human skin objects. Ultraviolet, visible and penetrative infrared images recorded by $\mathrm{CCD}$ cameras are used as the input data. A new genetic method based on mathematical morphology has been developed to detect objects and estimate shape properties of skin objects such as speckles and blood vessels on an arbitrarily chosen area. A very fast system could be achieved, and as no presumptions are used on the shape of the objects in question, the developed method is widely applicable to various kinds of images. The described transputer network was found to be very suitable for object analysis tasks due to its high performance and flexibility.
\end{abstract}

\section{Introduction}

Detection and investigation of external and internal skin objects originating from benign or malign skin deformities have a great significance not only in the field of dermatology but also for the cosmetic industry. However it is difficult the obtain appropriate data even by high cost systems like Computer Tomography and Magnetic Resonance Imaging on arbitrary skin areas in few $\mathrm{mm}$ depth. The reason for this is not only the low resolution but the difficulty and time requirement of obtaining cross sections in few $\mathrm{mm}$ wide intervals by these equipment. There are also instruments to provide pictures on a given spot or on a few $\mathrm{mm}$ wide skin area, like ultrasonic dermatology scanners etc. However, there is no system developed to provide automatic shape characteristics of skin objects of a large skin surface. It is not a simple task to determine objects and shapes within images, especially when the time requirements for this operation are severe. The developed techniques are rather specific for a given problem, and use computationally heavy methods for segmentation and pattern matching [1].

This paper presents a new method based on a genetic approach to detect objects and estimate shape properties (perimeter, area, mean diameter, shape factor) and distribution of skin objects like speckles and blood vessels on an arbitrarily chosen area. Our objective was to develop a fast system based on a parallel architecture that can provide global internal information about the human skin of the whole body or given parts of the body in 10 to $15 \mathrm{~mm}$ depth and to provide a fast method for the detection of the skin objects. 


\section{The optical and image acquisition system}

For the above purpose infrared, visible and ultraviolet images have been used. The infrared light, even in the near infrared band, where the wavelength is between 700 and $1000 \mathrm{~nm}$, can penetrate to 10 to $15 \mathrm{~mm}$ depth under the surface of the skin and the reflected part of this penetrating light can be recorded by CCD cameras. On the other hand, the ultraviolet light beam is totally reflected from the surface without any penetration into the subsurface layers of the skin. A schematic description of the light skin surface interaction is shown in Fig. 1.

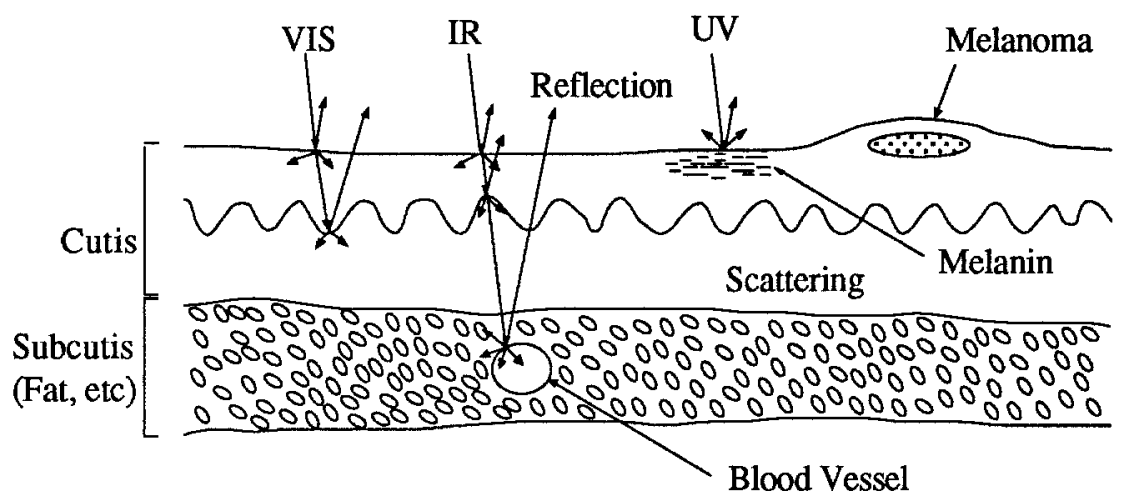

Fig. 1. Lightbeam and skin surface interaction

A CCD camera device, equipped with a mirror system has been developed. Our equipment consists of five CCD cameras, two for the ultraviolet band, filtered by 200-300 nm and 300-400 nm band filters, a visible camera for the $400-700 \mathrm{~nm}$ band and two CCD cameras for the infrared band extracted by $700-800 \mathrm{~nm}$ and $800-900 \mathrm{~nm}$ band filters. The light beam entering the camera system is first split into ultraviolet, visible and infrared bands on a mirror and filter system that is located in front of the cameras. The video signals produced by the camera system are digitized on an 8 bit, 10 Msampling/s analog/digital converter and stored in the memory of the main transputer. The total hardware layout is shown in Fig. 2.

\section{The transputer network}

The transputer network consists of two pipeline connected lattice networks, each of them containing sixteen T800 transputers. This architecture supports both algorithmic and data parallelization at the same time. The main transputer is also a T800 transputer with 4 Mbyte extended memory, while each subnode in the network has 1 Mbyte local memory. The main transputer is connected to the root transputer board located in the host Sun workstation through an RS422 interface. This host machine provides the user interface comprising the user control of the focus and iris, the selection of the Look Up Tables, booting the application into the network and displaying and post processing of the calculated images. 


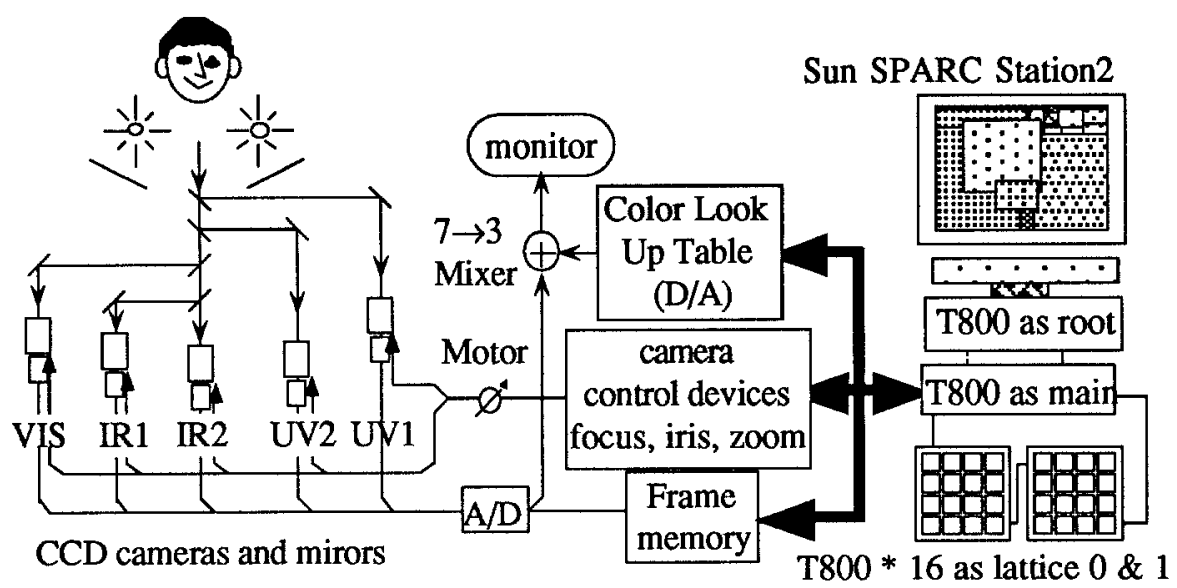

Fig. 2. Hardware layout

\section{The imaging method}

\subsection{Initialization}

For the detection of the skin objects, combinations of ultraviolet, visible and infrared band images are composed by using the camera device. First an arbitrary segmentation process is carried out by accomplishing separated two-dimensional Laplacian of Gaussian operation [2]. Object areas surrounded by edges are then decomposed for the determination of the morphological structuring element series representing the starting solution set for the genetic method. In this processing step, the separated consequent one dimensional calculation tasks are carried out parallel on the 32 transputer nodes by applying a careful data division, keeping overlapping data on the neighborhood nodes. Two horizontally adjacent nodes accomplish the separated LoG operation steps and then the resulting data are summed and saved on the appropriate transputer nodes.

\subsection{Genetic method}

Genetic algorithms were first introduced by Holland [3, 4] and implement evolutionary theories for solving nontrivial, mainly optimization problems. Here we use this approach to provide a new method for object detection. In our case a series of small size (3x3) structuring elements as shape composition blocks of the skin objects to be detected is acting as the element (chromosomes) of the genetic method. The starting structuring element series are determined on the basis of the arbitrarily segmented objects and used to create initial population. A series of morphological openings and closings is carried out by the elements of the operator sets. Then a binary encoding is accomplished for the generation of the initial population on the basis of the results obtained from the morphological operations. This can be 
accomplished by filling up strings from the $3 \times 3$ elements by preserving the $3 \times 3$ elements structure. Then we have to determine an evaluation value for each element. The evaluation value for each element of the population can be determined as the measure of fitness. This latter can be defined as the homogeneity of the region that can be calculated from weighed differences of pixel intensities of the umbra generated by the morphological operations with the given structuring elements. The morphological opening and closing operations can be implemented by very low level image operations [5] that are image shifting, union, and intersection of the generated sets because dilation and erosion can be expressed in the following way:
$\mathrm{A} \oplus \mathrm{B}=\bigcup_{\mathrm{b} \in \mathrm{B}} \mathrm{A}_{\mathrm{b}}$
$A \ominus B=\cap_{b \in B} A_{-b}$

where $A$ is the object set, and B is the structuring element set.

The above algorithm is accomplished on the transputer network by dividing the image into segments, such as each segment includes one or a few skin spots. We use an image scanning and boundary tracing task running on the main transputer to determine the data segments, and another one also running on the main transputer to provide these data for the transputer nodes by multidirectional data loading. The dilation and erosion steps are independent tasks on the nodes. In order to avoid the unbalanced processing on the network, data can be provided from a computationally heavy node to an idling one. It can be accomplished by registering independent tasks for local communication on the four serial links. These communication tasks can also handle the problem of providing external data for the shift operations on a given segment. After calculating the fitness values of the determined objects, finding regions fitting better to the image data is started by carrying out crossovers and some mutations on selected elements of the population that have relatively higher fitness values. The crossovers and mutations are accomplished on randomly paired elements by a given probability that is relatively low especially for mutation $(0.01)$. In our case, the crossovers are two dimensional operations. However, as they are restricted to horizontal and vertical splits, they can be implemented as crossovers of bitsequences. Tasks for carrying out the selection, crossover, and mutation can be accomplished parallel by alternating the processing and data getting and passing tasks by applying a proper timeout. A new offspring of the population can be obtained by the crossovers and mutations and the fitness values of the new elements have to be regenerated. Then a new processing loop can be started until either the required fitness is reached or no more progress can be measured. The average time required for the calculation on $512 \times 512$ size images is less than $350 \mathrm{~ms}$ applying 32 nodes for images containing 10 to 100 objects and using a population of 50 elements. However, this time is depending very much on the size and number of objects. If the number of objects is more than 100 the efficiency is significantly reduced due to the communication time required for the data reloading. The best fit shapes are then classified by determining the shape factors as the relation between the perimeter, the area, and the mean diameter of the regions. An actual implementations for a female face in Fig. 3.1. The pictures in the third line of this figure present the size and perimeter distributions and 

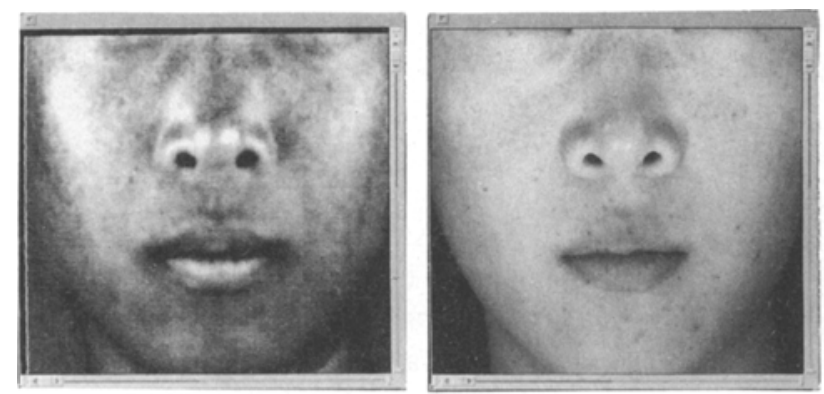

Ultraviolet image

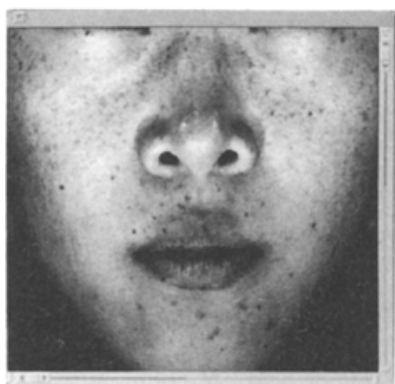

Input image

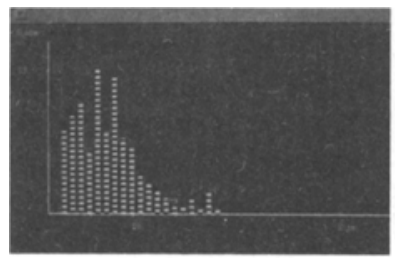

Mean size distribution

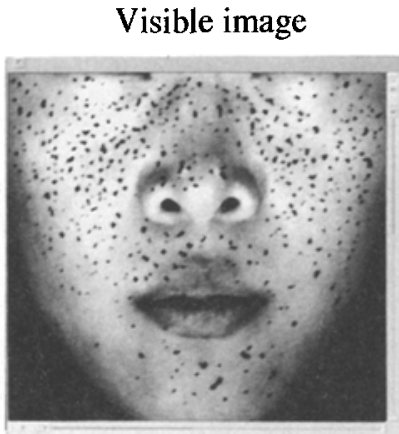

Detected spots

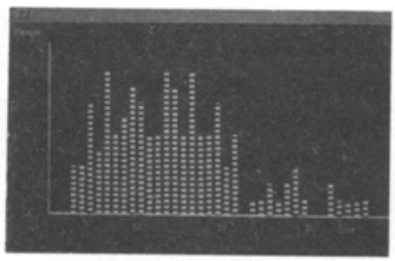

Perimeter distribution

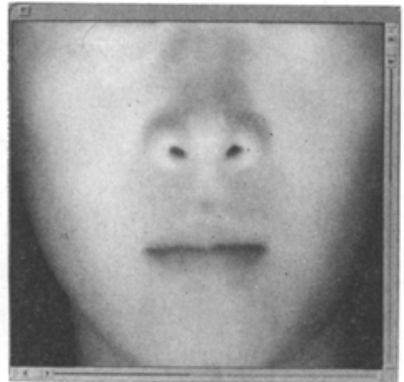

Infrared image

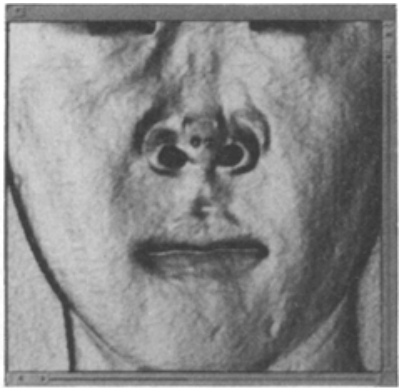

Shaded image

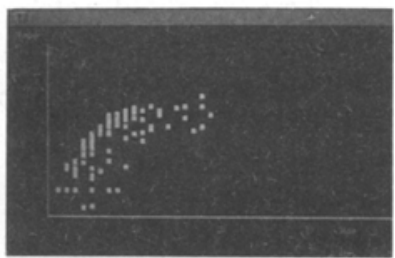

Shape factor correlation

Fig. 3.1. Skin spots on a female face (image size: $512 \times 512$ pixels)

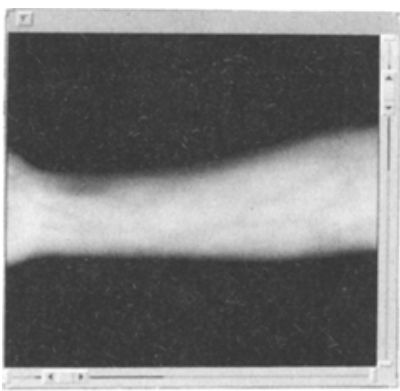

Input image

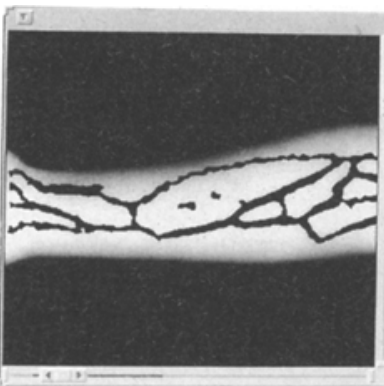

Detected blood vessels

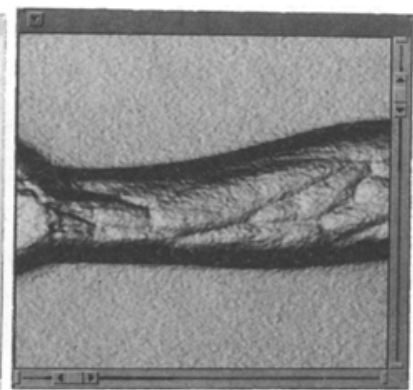

Shaded blood vessels

Fig. 3.2. Blood vessels on a female arm (image size: $512 \times 512$ pixels) 
the shape factor versus size correlation of the spots. Fig. 3.2. shows an application for blood vessel detection on a female arm.

\subsection{Network programming and user interface}

As a programming language, we have used OCCAM 2 for carrying out the algorithms on the transputer network as it generates an effective code [6]. The user interface is written in $\mathrm{C}$ on the host machine providing a convenient environment to the user in controlling and setting the cameras and LUTs, selecting the input picture for the calculation, starting calculation, displaying results. The latter functions are implemented as simultaneous light weight processes.

\section{Conclusions}

A new parallel system for low cost analysis of skin objects such as skin spots and blood vessels is discussed in this paper. A genetic algorithm has been developed on the basis of morphological structuring elements. A very fast system with about 15 times speedup can be achieved by the 32 transputer nodes as compared to the performance of the 28MIPS Sun workstation 2. Due to the flexibility and high performance of the transputers there are no constraints regarding on the size of the input images. However, the larger the input image is the less significant speedup can be achieved due to the communication overhead on the nodes caused by the reloading. Avoiding this problem is the object of further investigation. The developed method can be easily applied to wide areas of the image analysis field as no preliminary information is required about the objects to be determined.

\section{Acknowledgement}

The support for this research by Kyowa Hakko Kogyo Co., Ltd. is highly appreciated.

\section{References}

1. R. M. Haralick, L.G. Shapiro: Computer and robot vision. New York: Addison Wesley 1992

2. A. Huertas, G. Medioni: Detection of intensity changes with subpixel accuracy using Laplacian of Gaussian masks. IEEE Transactions on Pattern Analysis and Machine Intelligence 7, pp. 651-664 (1986)

3. J. Holland: Adaptation in natural and artificial systems. Ann Arbor: University of Michigan Press 1975

4. Z. Michailewicz: Genetic algorithms + data structures $=$ evolution programs. Berlin: Springer 1992

5. X. Zhuang, R. M. Haralick: Morphological structuring element decomposition. Computer Vision Graphics and Image Processing 35, pp. 370-382 (1986)

6. INMOS Limited: The transputer applications notebook, architecture and software, London: Prentice Hall 1989. 